\title{
Single Synaptic Vesicle Tracking in Individual Hippocampal Boutons at Rest and during Synaptic Activity
}

\author{
Edward A. Lemke and Jurgen Klingauf \\ Department of Membrane Biophysics, Max Planck Institute for Biophysical Chemistry, D-37077 Goettingen, Germany
}

\begin{abstract}
How synaptic vesicles move within central nervous synapses to their docking sites at the plasma membrane is widely discussed in synaptic physiology. This question is especially difficult to investigate in the small hippocampal boutons, which themselves can slowly move during observation in primary cell culture. Here, we describe a single particle tracking method using dual fluorescent dye labels that enabled us to visualize the movements of a single vesicle and the respective synaptic bouton simultaneously during resting conditions and stimulation. We found vesicle mobility to be very low in the absence of stimulation, in line with previous studies. Interestingly, mobility was also found to be low during synaptic activity. We found that vesicles labeled preferentially via early, late, and spontaneous endocytotic mechanisms behaved similarly at rest and during stimulation.
\end{abstract}

Key words: vesicle tracking; FM dyes; presynaptic mechanisms; vesicle mobility; vesicle recycling; endocytosis

\section{Introduction}

Central nervous synapses are packed with small synaptic vesicles that release their content only at specialized locations, the active zones (AZ), and have to be recycled locally thereafter (for review, see Sudhof, 2000; Matthews, 2004; Rizzoli and Betz, 2004). The mechanisms governing translocation of endocytic vesicles back to the active zone, and whether this involves diffusion or active transport, are essentially unknown.

Although electrophysiological techniques indirectly report pre-exocytotic events, such as vesicle movement inside the synapse (for review, see Korn and Faber, 1991), and electron microscopy provides static pictures (Heuser and Reese, 1973; Schikorski and Stevens, 1997; Harata et al., 2001a), real-time imaging and spectroscopic techniques seem to be ideally suited for this task. Imaging studies performed by Ryan (1999) suggested a role for myosin in vesicle cycling in hippocampal boutons. Conclusions, however, could be made only indirectly by inspecting the endoexocytotic recycling characteristics of synapses stained with fluorescent styryl dyes.

In the past, fluorescence recovery after photobleaching (FRAP) was used to probe vesicle mobility in several preparations (Henkel et al., 1996; Kraszewski et al., 1996; Holt et al., 2004; Rea et al., 2004), and Jordan et al. (2005) recently applied fluorescence fluctuation spectroscopy (FFS) to also study vesicle mobility in small synapses. In many of these studies, vesicles were se-

Received July 19, 2005; revised 0ct. 7, 2005; accepted 0ct.7, 2005.

This work was supported by the Deutsche Forschungsgemeinschaft Grant SFB 406 (J.K.), and E.A.L. is a scholar of the Boehringer Ingelheim Fonds. We thank Erwin Neher for his guidance and support throughout this work and Takeshi Sakaba for critically reading this manuscript. We are grateful to Johann Engelhardt and Stefan Hell for retrofitting the laser-scanning microscope with a photon counting chip.

Correspondence should be addressed to Dr. Jurgen Klingauf, Department of Membrane Biophysics, Max Planck Institute for Biophysical Chemistry, D-37077 Goettingen, Germany. E-mail: jklinga@gwdg.de.

E. A. Lemke's present address: Department of Chemistry and Department of Molecular Biology, The Scripps Research Institute, 10550 North Torrey Pines Road, La Jolla, CA 92037.

DOI:10.1523/JNEUROSCI.2971-05.2005

Copyright $\odot 2005$ Society for Neuroscience 0270-6474/05/2511034-11\$15.00/0 lectively labeled using fluorescent styryl dye (FM dyes) (Betz et al., 1992). These dyes, however, are released during synaptic activity, limiting FFS and FRAP studies mainly to the steady state. In endocrine cells, single-particle tracking emerged as a powerful tool to study secretory granule dynamics (Steyer and Almers, 1999; Levitan, 2004). Here, individual large secretory granules can be easily imaged using evanescent wave microscopy. Unfortunately, in central nervous synapses, vesicles are much smaller and densely packed, such that individual vesicles cannot be distinguished if several vesicles are fluorescently labeled (but see Aravanis et al., 2003; Gandhi and Stevens, 2003). Furthermore, the investigation of vesicle movement can be compromised by synapse movement itself (Fischer et al., 1998; Dunaevsky et al., 1999). Thus, it is not surprising that the fundamental question on how synaptic vesicles move within the cluster to the active zone is still a matter of debate. Only in giant bipolar cell terminals of the goldfish retina was it possible to track single synaptic vesicles using evanescent wave microscopy (Zenisek et al., 2000). This technique, however, is limited to preparations in which the presynaptic terminal can be isolated and attached to a glass coverslip, which is currently not possible for small central nervous synapses. Furthermore, in contrast to ribbon synapses (Zenisek et al., 2000; Holt et al., 2004), synaptic vesicles in hippocampal boutons seem to have very low mobility (Kraszewski et al., 1996; Jordan et al., 2005), pointing toward a more organized vesicle cluster.

In this study, we describe the successful application of singleparticle tracking (Saxton, 1993) to the study of single synaptic vesicles in individual presynaptic boutons in the absence of and during weak stimulation. We first outline the problems that, so far, precluded single vesicle tracking (SVT) in small synaptic boutons, and describe a dual-color approach using a highly sensitive detection unit mounted on a laser-scanning microscope to tackle them. In the second part, we apply this technique to gain insights into the dynamics of single synaptic vesicles endocytosed in response to different stimulation paradigms. 


\section{Materials and Methods Primary hippocampal cell culture}

Dissociated hippocampal cultures were prepared from 1- to 3-d-old Wistar rats according to previous protocols (Klingauf et al., 1998). Neurons were used for experiments after 14-20 d in vitro.

\section{FM dye loading}

Coverslips with neurons growing on them were mounted in a perfusion chamber of an inverted microscope (TCS SP2; Leica, Wetzlar, Germany). Synapses were stained by eliciting action potentials in the culture in the presence of fluorescent styryl dyes (FM dyes) using electric field stimulation. One millisecond current pulses of $40 \mathrm{~mA}$ were delivered with alternating polarity by two platinum electrodes spaced at $1 \mathrm{~cm}$ on the bottom of the chamber. Cells were perfused by a modified Tyrode's solution containing the following (in $\mathrm{mM}$ ): $150 \mathrm{NaCl}, 5 \mathrm{KCl}, 2 \mathrm{CaCl}_{2}, 1$ $\mathrm{MgCl}_{2} 10$ HEPES, pH 7.4, 30 glucose. To block recurrent activity, $10 \mu \mathrm{M}$ CNQX and $50 \mu \mathrm{M}$ AP-5 (Tocris, Northpoint Forthway, UK) were added.

To achieve fluorescent double labeling in red and green of synaptic boutons, the protocol shown in Figure $1 a$ was used. First, the recycling pool of the synapses was stained with the red fluorescent dye FM5-95 by application of 600 action potentials (APs) delivered at $20 \mathrm{~Hz}$ during a $60 \mathrm{~s}$ presence of a low concentration (2.5 $\mu \mathrm{M})$ FM5-95 (Harata et al., 2001b). Cells were then washed for $10 \mathrm{~min}$ in a medium containing only minimal $(0.1 \mathrm{~mm}) \mathrm{Ca}^{2+}$. Next, synapses were labeled with a high concentration of FM1-43 (16 $\mu \mathrm{M})$ using a minimal-staining protocol to stain only a few vesicles per synapse. The three different minimal-staining protocols used are described below.

\section{Early endocytosed vesicles}

Five APs were applied at $5 \mathrm{~Hz}$ during a 10 s presence of FM1-43 dye.

\section{Late endocytosed vesicles}

One hundred APs were applied at $10 \mathrm{~Hz}$. Thirty seconds after stimulus end, the cells were perfused with FM1-43 dye for $10 \mathrm{~s}$.

\section{Spontaneously endocytosed vesicles}

FM1-43 was applied for $20 \mathrm{~s}$ in the absence of any stimulation but in elevated $\mathrm{Ca}^{2+}$ solution ( $8 \mathrm{~mm}$ instead of $2 \mathrm{~mm}$ ). After washing of the cells in minimal calcium solution, the actual image collection started (see below) in modified Tyrode's solution with $2 \mathrm{~mm} \mathrm{Ca}^{2+}$.

At the end of the experiments, $600 \mathrm{APs}$ were applied two times at $20 \mathrm{~Hz}$ to fully destain the synapses.

\section{Laser-scanning microscopy setup with custom-build detection unit}

Images were acquired with a Leica SP2 confocal microscope equipped with a $63 \times / 1.2 \mathrm{~W}$ objective. The $\lambda=488 \mathrm{~nm}$ wavelength of an argon laser was used for illumination. The emission light was focused on a pinhole in the conjugated image plane (set to 1.7 Airy units) and directed to an external detector unit. Fluorescent light was split into green and red channels using a 610 DCXR dichroic mirror (AHF, Tuebingen, Germany). A bandpass filter at $\lambda$ of $510-560 \mathrm{~nm}$ and a long-pass filter at $\lambda$ of $>630 \mathrm{~nm}$ (AHF) were used to maximize the collection efficiency of green fluorescent light by having only a minor bleed-through of red fluorescent light into this detection channel (3\% red in green) and vice versa (15\%). To reduce nonlinear detection effects of the avalanche photo diodes (APDs) [green channel, SPCM-AQR-13 (PerkinElmer, Rodgau, Germany); red channel, SPCM-AQ-231 (EG\&G, Vaudreuil, Quebec, Canada)], the emission light was split in each detection channel with a R50/T50 mirror (AHF) and focused onto the APDs. The transistor-transistor logic pulses from the APDs were shortened to $6 \mathrm{~ns}$, and the counts of each detection channel were combined using a custom-made pulse combiner (PicoQuant, Berlin, Germany) and counted. We determined the minimal waiting time between two photon detection events (dead time) for each channel to be $t_{d} \approx 30 \mathrm{~ns}$.

\section{Image acquisition}

The image acquisition process was divided into z-stack and time-series recordings (see Fig. $1 a, b$ ). $z$-stack recording

A z-stack of 10 images spaced at $\approx 0.4 \mu \mathrm{m}$ was acquired at the beginning and at the end of every experiment to allow for off-line analysis (see below) of the absolute intensities of red and green fluorescent puncta before and after the destaining stimulus. The pixel dwell time was kept constant to $4 \mu \mathrm{s}$, and the sample was scanned unidirectionally.

\section{Time-series recording}

Between the two z-stack recordings, a time series was acquired that was used for off-line single-particle tracking analysis (see below). Fast acquisition of each image $(1024 \times 1024$ pixels; 1 pixel $=0.058 \mu \mathrm{m})$ was achieved in bidirectional-scanning mode with a constant pixel dwell time of $0.5 \mu \mathrm{s}$. The laser power $(\approx 19 \mu \mathrm{W}$ at the back focal plane of the objective) was increased eightfold, relative to the $\mathrm{z}$-stack recording, to match fluorescence intensities. For tracking vesicle motion during resting conditions and synaptic activity, the stimulation protocol shown in Figure $1 b$ was time-aligned with image acquisition. First, 15 images were acquired with a scan time of $1.512 \mathrm{~s}$ and no lag between images. Then, the culture was stimulated for $22.5 \mathrm{~s}$ at $3 \mathrm{~Hz}$ while 15 more images were acquired. The stimulation frequency was then increased to $20 \mathrm{~Hz}$, and another 10 images were collected. The last five images were spaced at $1.5 \mathrm{~s}$. Bleaching of vesicular FM1-43 in the fast-acquisition mode was determined to have a half-time ( $\left.t_{1 / 2}\right)$ of 122 frames (data not shown), and this was corrected for in the data analysis assuming bleaching to be monoexponential.

Our detectors counted the number of photons arriving during a given pixel dwell time, and all intensities in this study are given in counts.

\section{Data analysis}

Reproducible single vesicle tracking must fulfill the following two requirements: (1) only single vesicles with similar fluorescence intensity should be selected and (2) because every single particle algorithm has a precision limit given by the signal-to-noise ratio in the region of interest (ROI), only single vesicles in regions of similar image quality should be tracked.

To fulfill these requirements, we developed a semiautomated algorithm, which we describe below. The routine applied selection criteria, which we divided into those applied to the time series and those to the Z-stack recording. Although the time series allowed one to check whether the signal-to-background ratio was sufficient in every image used for particle tracking, the object's absolute intensity might be underestimated if it was not perfectly in focus. Because the absolute intensity was the crucial parameter to confirm the presence of only one vesicle in the region of interest, the intensity of all fluorescent objects was measured in $\mathrm{z}$-stack recordings acquired before and after time series acquisition. This allowed us to calculate the difference in fluorescence intensity $(\Delta F)$ loss caused by stimulation in an ROI, by calculating the difference in the integrated intensity in an ROI in the plane of best focus before and after stimulation.

Intensity analysis of green and red fluorescent puncta from z-stack recordings. In previous protocols, single vesicles were identified by first mildly staining synapses using a weak stimulation protocol; in a second step, strong staining was performed to identify functional synapses (Ryan et al., 1997; Murthy and Stevens, 1998; Aravanis et al., 2003; Jordan et al., 2005). Both rounds were performed using only one fluorescent dye. In our study, only one round of data acquisition was necessary to identify single vesicles and synapses. ROIs were defined as those in which a bright red fluorescent spot $(\Delta F>100$ counts, integrated over a square region with a $1.1 \mu \mathrm{m}$ edge length) could be identified. All $\Delta F$ intensities were the intensity differences before and after the destaining stimulus in the plane of best focus of the z-stack recording. In the same ROI, the fluorescence was measured in the green channel by integration over a square region (edge length, $0.62 \mu \mathrm{m}$ ) centered on the green spot (see below for details) and was plotted in a histogram, which, in the green channel, typically showed a multimodal distribution of equidistant peaks. Histograms, as in Figure $2 f$, were analyzed similarly, as described by Murthy and Stevens (1998) and Jordan et al. (2005). The width of each peak in the histogram was a sum of the measuring error and the variability in the size of vesicles. The measurement error was the sum of the errors in the intensity mea- 
surement of the two images (before and after the destaining stimulus), which was $c_{m}^{2}\left((\mu \times k+r)^{2}+r^{2}\right)$, with $\mu$ being the intensity (in detected counts) of the $k$ th peak; $r$, the residual fluorescence after complete destaining, which was found to be approximately constant $(r=40$ counts); and $c_{m}$, the coefficient of variation for the intensity measurement. This coefficient was determined by repeated measurements of green fluorescent spots and was found to be approximately constant with $c_{m}=0.08$. The coefficient of variation in vesicle size was adapted from Murthy and Stevens (1998) to be $c_{v}=0.2$. The error resulting from size variations was given by $c_{v}^{2} \times \mu^{2} \times k$. The quantal histogram with three peaks could be fit with the following equation:

$$
\sum_{k=1}^{3} A_{k} \times \exp \left(-\frac{1}{2} \times \frac{\left[x-\left(\mu \times k+\mu_{\text {offset }}\right)\right]^{2}}{c_{m}^{2}\left[(\mu \times k+r)^{2}+r^{2}\right]+c_{\nu}^{2} \times \mu^{2} \times k}\right) .
$$

The only fit parameters were the amplitude of each peak $A_{k}$ and the peak spacing $\mu$, which corresponds to the center of the first peak $(k=1)$ (i.e., the average fluorescence contribution of a single vesicle). The intensityoffset parameter, $\mu_{\text {offset }}$ of the parameter $\mu$, was found to originate from the minor bleed-through of red fluorescent light into the green detection channel (see Results). This contribution was determined by measuring the intensity in those ROIs in which a bright fluorescent spot was identified in the red channel, but no punctum in the green detection channel (absolute intensity of the green fluorescent spot at the beginning of the experiment, $<60$ counts).

Only ROIs in which a fluorescent spot belonging with $95 \%$ confidence to the first peak of the single vesicle histogram and $\Delta F>60$ counts to ensure high enough precision of the tracking algorithm (see next paragraph) were taken for analysis.

Off-line analysis of time-series recordings in the green channel for single vesicle tracking. In the green channel, images of the time series were first filtered using a Hanning bandpass filter (for details on filtering, see supplemental Fig. 1, available at www.jneurosci.org as supplemental material) to remove high-frequency noise and large background structures that lowered the precision of the tracking algorithm. For single vesicle tracking, a simple two-dimensional (2D) Gaussian was fit to a green fluorescent spot using a $\chi^{2}$-routine implemented in IgorPro (Wavemetrics, Lake Oswego, OR) (supplemental Fig. 2, available at www.jneurosci.org as supplemental material) as follows:

$$
\text { Gauss }_{2 \mathrm{D}}=A_{\text {offset }}+A \times \exp \left(-\frac{1}{2}\left[\frac{\left(x-x_{\mathrm{pos}}\right)^{2}}{\sigma_{x y}^{2}}+\frac{\left(y-y_{\mathrm{pos}}\right)^{2}}{\sigma_{x y}^{2}}\right]\right),
$$

with $A_{\text {offset }}$ being the offset of the amplitude $A$, and $x_{\text {pos }}$ and $y_{\text {pos }}$ the center coordinates of the Gaussian with width $\sigma_{x y}$. Two-dimensional Gaussian fitting is a convenient, easy to implement, and well characterized method to determine with high precision the position of a subresolution fluorescent particle (Cheezum et al., 2001; Thompson et al., 2002). In this study, vesicles stained under different biological conditions were analyzed and compared. For this to be possible, all vesicles taken for data analysis had to have similar signal-to-background ratios. This ratio was not easily accessible from small ROIs in an image. However, the parameters returned from the Gaussian fit could be used to ensure that vesicles were selected in a reproducible manner.

All of the following selection criteria were a compromise between selecting for those regions that allowed for tracking single vesicles with a high precision and what was experimentally feasible. More stringent selection criteria would result in higher precision but less data to analyze.

(1) The maximum offset for the 2D Gaussian fit, a measure of background, was 0.3 counts. This criterion had to be fulfilled in every image of the time series. This selects for regions with minimal background fluorescence.

(2) Only vesicles with an average amplitude of $>1.4$ counts during resting condition were tracked, to allow for high tracking precision (see Results). a

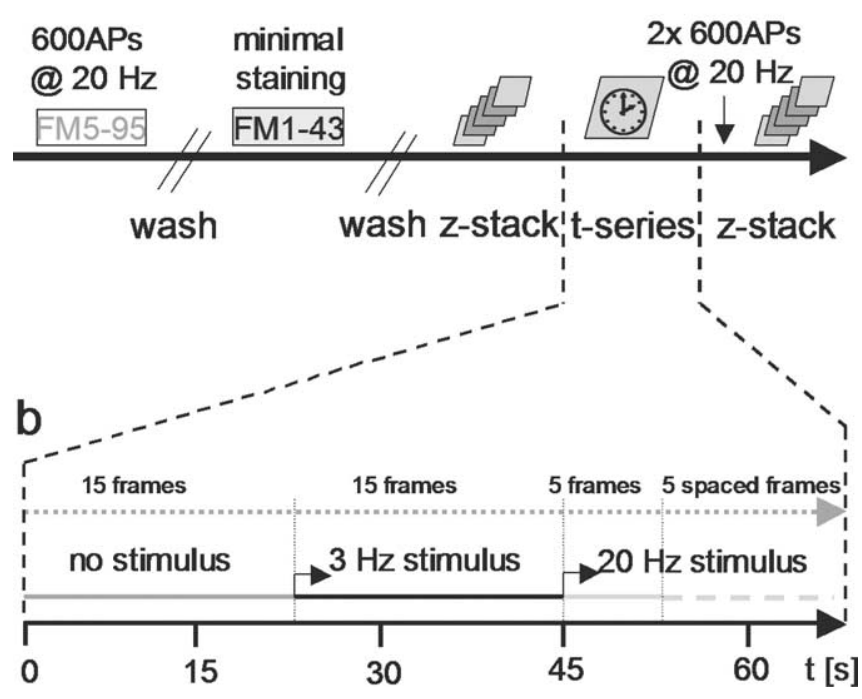

Figure 1. Experimental protocol. $\boldsymbol{a}$, Schematic representation of the dye loading protocol. First, the recycling pool of synapses was stained using $600 \mathrm{AP}$ at $20 \mathrm{~Hz}$ during 1 min application of $2.5 \mu \mathrm{m}$ low-concentrated FM5-95. The culture was washed for $10 \mathrm{~min}$ in minimal $\mathrm{Ca}^{2+}$ solution before a minimal staining protocol was applied using the green fluorescent FM1-43. Different minimal staining protocols were used in this work and are explained in Materials and Methods. Then, a z-stack of images was recorded, followed by an acquisition of a time series used for off-line tracking. After a strong destaining stimulus, a last z-stack recording was performed. $\boldsymbol{b}$, During the time series, APs were triggered after frame 15 (22.5 s) at $3 \mathrm{~Hz}$ for $22.5 \mathrm{~s}$ (15 frames). After frame 30, the stimulation frequency was increased to $20 \mathrm{~Hz}$. The last five frames were spaced at $1.5 \mathrm{~s}$.

(3) The image of a vesicle should be comparable with that of the point-spread function of the microscope. To determine the latter, $40 \mathrm{~nm}$ green fluorescent beads were allowed to settle on a neuronal cell layer and their width (in the filtered images) was determined by Gaussian fit to be full-width at half-maximum $(\mathrm{FWHM})_{x y}=0.383 \pm 0.055 \mu \mathrm{m}$. Please note that the imaging quality of vesicles was lowered by background fluorescence relative to beads, which lowers the quality of the fit. The only objects that were tracked were those whose width in the fit in every single image never exceeded a FWHM of $0.616 \mu \mathrm{m}$. Also, this criterion had to be fulfilled in every image of the time series. Lowering the threshold to $0.547 \mu \mathrm{m}$, for example (i.e., $0.383 \cdot 3 \times 0.055$ ), would remove $\sim 10 \%$ of the data from this work.

Off-line analysis of time-series recordings in the red channel for synapse tracking. One major problem in tracking small single vesicles was that the synapse itself could also move (Fischer et al., 1998). Our experimental design offered an easy possibility to ensure that only vesicle movement was analyzed by also tracking the synapses in the red detection channel. Strong staining of the recycling pool with FM5-95 resulted in larger fluorescent spots that were typically not subresolution anymore, but originated from larger vesicle clusters. Those structures were tracked in a square region (edge length, $1.7 \mu \mathrm{m}$ ) using a cross-correlation algorithm (for details, see supplemental Fig. 3, available at www.jneurosci.org as supplemental material) (Heintzmann, 1999). ROIs in which a shift of the red fluorescent spot of $>0.12 \mu \mathrm{m}$ was detected were always rejected from analysis (a correction for the shift was never performed). Note that such shifts could be a consequence of the low precision of the algorithm or movement of the bouton. Smaller bouton movement would only lower the precision of the single vesicle tracking experiments. However, this way, the influence of any residual bouton movement had a constant contribution to the tracking precision of green fluorescent spots in all experiments. Because we could not detect any difference in the mobility of resting vesicles and those in cultures fixed with paraformaldehyde, we conclude that our data on single vesicles were not contaminated by any residual bouton movement when using this strict selection criterion (see Results and Fig. 4 on fixed vesicles). 

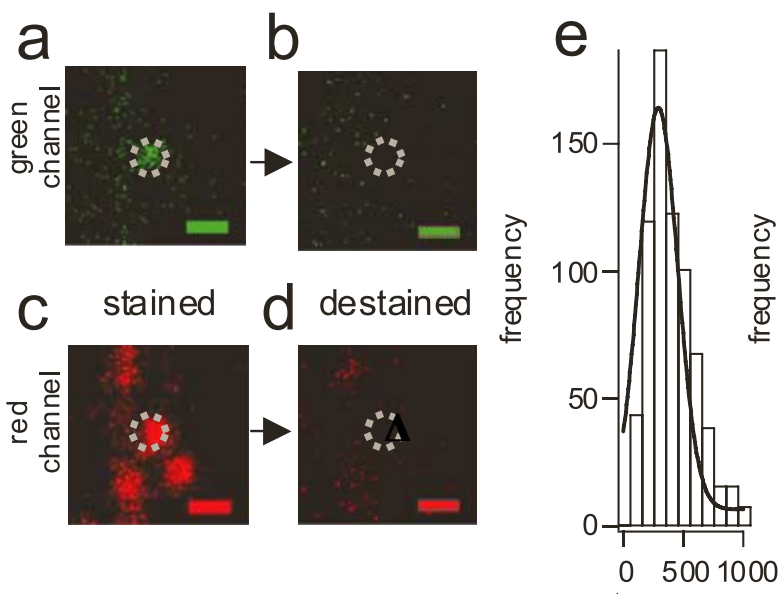

$\Delta \mathrm{F}[\mathrm{counts}]$

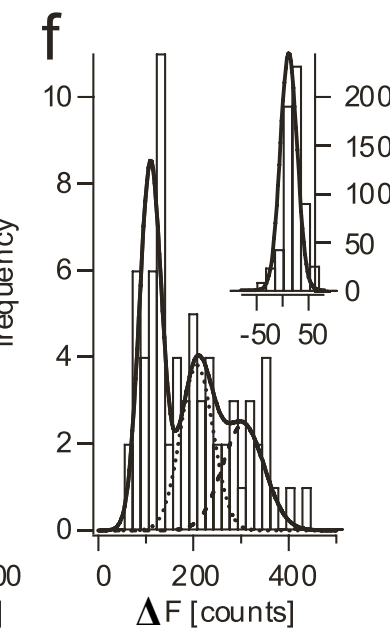

Figure 2. Minimal staining of early endocytosed vesicles. $\boldsymbol{a}$, Exemplar image of a putative single FM1-43-stained vesicle in an active synapse. Shown is the plane of best focus from a z-stack acquired after dual-color staining of the culture. The dim green fluorescent spot lost its fluorescence after strong stimulation $(\boldsymbol{b})$. $\boldsymbol{c}$, The same ROl was imaged in the red detection channel, in which synapses appeared as bright objects that released their fluorescence during stimulation (d). Scale bars, $1 \mu \mathrm{m} . \boldsymbol{e}, \Delta F$ intensities from calculating the differences between $c$ and $\boldsymbol{d}$. The mean synapse fluorescence intensity was obtained by a Gaussian fit yielding a $\Delta F$ of 294 counts. $f, \Delta F$ intensities for the green channel of $n=70$ synaptic boutons were plotted resulting in a multimodal distribution. The histogram could be fit with the quantal model given in Equation 1 yielding an intensity of a single vesicle of 99 counts. The dotted and dashed lines highlight the peaks for two and three stained vesicles per bouton, respectively. The inset shows the background intensity from bleed-through, determined to be 12 counts.

\section{Semiautomated algorithm for reproducible particle tracking under} different physiological conditions

To ensure that fluorescent puncta are always picked in a reproducible manner, we aimed at minimizing any bias by the experimenter and used a semiautomated algorithm as follows.

(1) The user selected spot-like structures in the raw data.

(2) To further select spot-like structures, the time-series selection criteria were applied to the first 15 frames of the time series. Because no stimulus protocol was applied during this period, the intensity of a vesicle was expected to be constant throughout this time.

(3) Next, the intensity of those ROIs were analyzed from the z-stack recording. Only those ROIs passing the aforementioned z-stack criteria were taken for analysis.

(4) Next, whole time series were analyzed by tracking the position of the object by two-dimensional Gaussian fitting. As explained in Results, a dye release event was determined by monitoring the amplitude of the Gaussian fit. The only ROIs that were pooled for final data analysis were those in which all frames until one frame before the detected dye-loss event (for explanation, see Results) fulfilled the time-series selection criteria, including the requirement that no synapse movement could be detected.

\section{Jump frequency distribution analysis}

Jump frequency distribution analysis was used to characterize the mobility obtained from single-particle tracking data (Crank, 1975). In this analysis, the jump distance between $\Delta n$ frames is plotted in a histogram and can be analytically fitted with the following equation:

$$
p\left(r_{\text {data }}, t\right) d r=\sum_{j=1}^{k} \frac{M f_{j}}{2 D_{j} t} e \frac{-r_{\text {data }}^{2}}{4 D_{j} t} r_{\text {data }} d r,
$$

to yield $D_{j}$, the two-dimensional diffusion coefficient for species $j$, with $t$ being the lag time between $\Delta n$ frames; $M$, the number of jumps analyzed; $f_{j}$, the fraction of species $j$ with $\sum_{i=0}^{\infty} j_{i}=1 ; r_{\text {data }}$, the jump distance; and $d r$, the analyzed jump interval (the bin size in the histogram plot) (see Results).

\section{Mean square displacement}

The mean square displacement was calculated as reported previously (Qian et al., 1991; Saxton, 1993; Steyer and Almers, 1999).

\section{Akaike information criterion}

Data fitting is typically done by maximizing the log likelihood of a model. An inherent problem to this process is that using more parameters to fit the data typically allows for increased quality of the fit, which, however, also increases the variance in each parameter. One convenient statistical tool to determine the model that best describes the data is the Akaike information criterion (AIC) (Akaike, 1981). The general equation for the AIC consists of two terms as follows:

$$
\begin{aligned}
\text { AIC }=-2 \times \log [\text { likelihood }(\text { model }) & +2 \\
& \times n .
\end{aligned}
$$

In the first term, the logarithm of the likelihood is calculated, which, as mentioned above, might return higher values using more complex models. Most importantly, the second term penalizes for the numbers of parameters $n$ used by the model. The value of the AIC is, thus, a number that is associated with every model. Relative comparison of the AIC values for different models allows for selection of the best one (i.e., the one with the lowest AIC value). For applying the AIC to the jump frequency distribution, we use Equation 3 by inserting the fitted parameters for $D_{j}$ and $f_{j}$ (for clarity, we hereafter write $D_{j}^{\text {fit }}$ and $\left.f_{j}^{\text {fit }}\right)$ and summing over all points the logarithms of the likelihood for each point $\left(r_{\operatorname{data}(l)}\right)$. Equation 4 then becomes the following equation:

$$
\mathrm{AIC}=-2 \times \sum_{l=0}^{l=M} \log \left[\sum_{j=1}^{k} \frac{M f_{j}^{\mathrm{fit}}}{2 D_{j}^{\mathrm{fit}} \times t} e^{\frac{-r_{\mathrm{dat}(l)}^{2}}{4 D_{j}^{\mathrm{fit}} \times t} r_{\text {data }(l)}} d r\right]+2 \times k .
$$

\section{Results}

If it was possible to monitor a single vesicle within an individual bouton over time, much information could be gained about the fundamental processes of vesicle dynamics. If it would be further possible to specifically investigate vesicles belonging to different vesicle pools, it should be possible to deepen our understanding on synaptic vesicle cycling. For this, it would be necessary to first label individual vesicles and track their movement over time, which might seem simple but has several major pitfalls.

One major problem arose from the fact that we aimed at studying the mobility of a $0.040 \mu \mathrm{m}$ subresolution object within a single bouton that measured only $\varnothing \sim 1 \mu \mathrm{m}$ (Schikorski and Stevens, 1997) and that itself could undergo slow movements (Fischer et al., 1998).

In general, the precision of every tracking algorithm depends on the signal-to-noise ratio in the image and is larger if only one object is present in an ROI. A single vesicle per synapse could be stained using FM dyes applied briefly during minimal stimulation (Ryan et al., 1997; Murthy and Stevens, 1998; Aravanis et al., 2003; Jordan et al., 2005). Unfortunately, staining a culture with FM dyes did not result only in stained vesicles, but also in unspecific staining of dead membrane structures and the astrocyte layer, causing spatial variations in the signal-to-noise ratios. Reproducible particle tracking thus required an algorithm that selected ROIs with comparable imaging quality.

Another problem in single synaptic vesicle tracking arose from the fact that the intensity of single vesicles stained with FM 

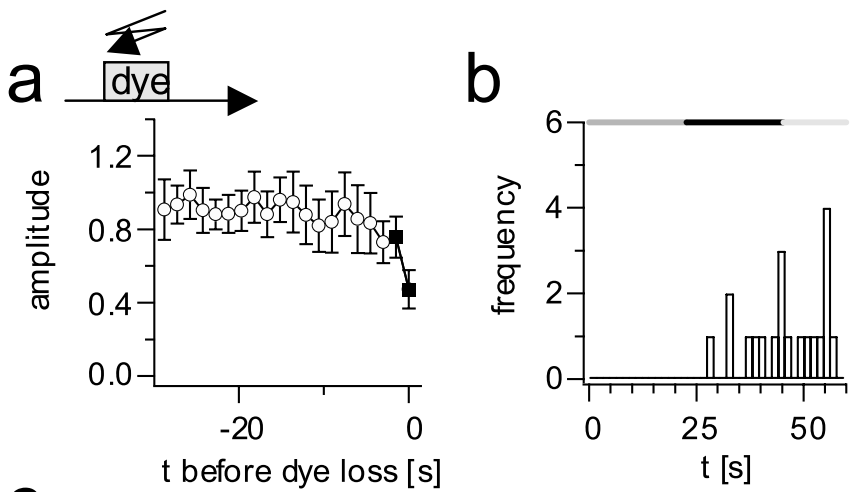

C

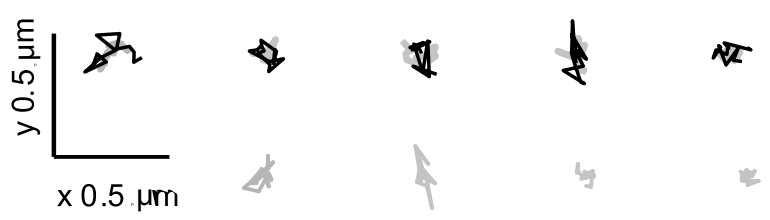

Figure 3. Qualitative analysis of early endocytosed vesicles. $\boldsymbol{a}$, Fluorescence amplitude time series of single vesicles, aligned to the time of dye loss (at $t=0 \mathrm{~s}$ ) for all vesicles for which a dye-loss event, until frame 35 , was detected ( $n=15$ ). Shown are the amplitudes returned from the Gaussian fits. The last data point before dye loss and the one in which the dye loss was detected are highlighted in black, because they were typically excluded from mobility analysis (see also Fig. 4). $\boldsymbol{b}$, Shown is the distribution of dye loss events during the time series. Colored bars on top indicate the stimulus protocol (see also Fig. 1b). c, Shown are five exemplar trajectories of individual vesicles in the top row. Trajectories are plotted in gray during resting conditions and in black lines during $3 \mathrm{~Hz}$ stimulation. In the bottom row, four trajectories of vesicles in a culture fixed with $4 \%$ paraformaldehyde are shown. Please note that $0.5 \mu \mathrm{m}$ corresponds approximately to one-half the diameter of a synapse. Error bars indicate SEM.

dyes during acquisition of the time series changed if a stimulus protocol was applied simultaneously. Dye was then released maybe fully or partially from the vesicle (Henkel and Betz, 1995; Klingauf et al., 1998; Aravanis et al., 2003). Furthermore, dye loss was not necessarily an instantaneous process because of the departitioning rate of these dyes (Henkel and Betz, 1995; Ryan et al., 1996a; Klingauf et al., 1998). If a major portion of dye, but not all, was lost or had not yet left the ROI, the precision of the tracking algorithm could decrease. For proper analysis of vesicle mobility during stimulation, one had to eliminate those data from the analysis.

In the following, we describe how we solved these major problems and then present data on single vesicle tracking, first for vesicles that were endocytosed early, and then for vesicles endocytosed in a late step and spontaneously.

\section{Addressing problem 1: simultaneous imaging of single vesicles and corresponding synapses}

Protocols using one fluorescent dye to stain single vesicles in boutons were used previously to study properties of synaptic function on the single vesicle level other than mobility (Ryan et al., 1997; Murthy and Stevens, 1998; Aravanis et al., 2003). Recently, we determined the fluorescence intensity contribution of a single vesicle on our setup using a one-color staining protocol using FM1-43 dye (Jordan et al., 2005). Taking slightly different optical characteristics (e.g., laser intensity, emission filter) into account, we expected the fluorescence intensity of a single vesicle in the following experiments to be $\Delta F \approx 100$ counts. We modified the one-color protocol and established a dual-color staining protocol, making use of the spectrally separable styryl dyes FM5-95 (red) and FM1-43 (green). As outlined in Figure 1 $a$, the culture was first stained strongly with FM5-95, washed, and then stained minimally using electrical stimulation with $5 \mathrm{AP}$ at $5 \mathrm{~Hz}$ during a $10 \mathrm{~s}$ application of $16 \mu \mathrm{M}$ FM1-43, which preferentially stained vesicles endocytosed during or immediately after the stimulus (Ryan et al., 1996a, 1997). We will refer to vesicles stained using this stimulation protocol as early endocytosed vesicles. A z-stack recording was performed before and after application of a strong destaining stimulus (Fig. 1a) and used for offline intensity measurements in the plane of best focus. As shown in Figure $2 c$, synapses appeared as bright red fluorescent structures in the red channel, although for some synapses, spots were also observed in the green detection channel (Fig. $2 a$ ). The fluorescence from those objects diminished after stimulation of the culture (Fig. $2 b, d$ ). In Figure $2 e$, the distribution of $\Delta F$ fluorescence intensities for ROIs in the red detection channel is shown, with a mean intensity of 292 counts corresponding to $\sim 50$ vesicles, as we determined previously (Jordan et al., 2005). For the green channel (Fig. 2f), the multimodal histogram yielded a distribution of three peaks. This histogram was very similar to those

Table 1. Summarized are the results from vesicle tracking experiments on early endocytosed vesicles, late endocytosed vesicles, and spontaneously endocytosed vesicles, as well as from cultures treated with $4 \%$ paraformaldehyde (PFA)

\begin{tabular}{|c|c|c|c|c|c|}
\hline \multirow[b]{2}{*}{ Parameter } & \multirow[b]{2}{*}{ Unit } & \multicolumn{3}{|l|}{ Endocytosis type } & \multirow[b]{2}{*}{ PFA } \\
\hline & & Early & Late & Spontaneous & \\
\hline$n$ green fluorescent spots & & 59 & 161 & 76 & 81 \\
\hline Intensity single vesicles & Counts & 98 & 99 & 113 & \\
\hline n"trackable" single vesicles & & 20 & 21 & 22 & 22 \\
\hline Dye loss during first 75 APs & $n$ & 10 & 10 & 11 & \\
\hline Synapse intensity after 75 APs & $\%$ & 78 & 84 & 77 & \\
\hline \multicolumn{6}{|c|}{ Jump frequency analysis for vesicles under resting conditions } \\
\hline$n$ frames resting conditions & & 208 & 218 & 223 & 252 \\
\hline$D^{\text {nostim }}$ & $\mu \mathrm{m}^{2} / \mathrm{s}$ & $4.31 \times 10^{-4}$ & $5.33 \times 10^{-4}$ & $4.94 \times 10^{-4}$ & $4.70 \times 10^{-4}$ \\
\hline \multicolumn{6}{|c|}{ Jump frequency analysis for vesicles during stimulation } \\
\hline One-component analysis & AIC & -775 & -772 & -801 & \\
\hline$D^{\text {stim }}$ & $\mu \mathrm{m}^{2} / \mathrm{s}$ & $9.312 \times 10^{-4}$ & $7.20 \times 10^{-4}$ & $5.78 \times 10^{-4}$ & \\
\hline Two-component analysis & AIC & -1063.85 & -974 & -989 & \\
\hline Fraction $f_{1}^{\text {stim }}$ & $\%$ & 22 & 81 & 48 & \\
\hline$D_{1}^{\text {stim }}$ (fixed to $D^{\text {nostim }}$ ) & $\mu \mathrm{m}^{2} / \mathrm{s}$ & $4.31 \times 10^{-4}$ & $5.33 \times 10^{-4}$ & $4.94 \times 10^{-4}$ & \\
\hline Fraction $f_{2}^{\text {stim }}$ & $\%$ & 78 & 19 & 52 & \\
\hline$D_{2}^{\text {stim }}$ & $\mu \mathrm{m}^{2} / \mathrm{s}$ & $11.61 \times 10^{-4}$ & $19.41 \times 10^{-4}$ & $30.22 \times 10^{-4}$ & \\
\hline
\end{tabular}



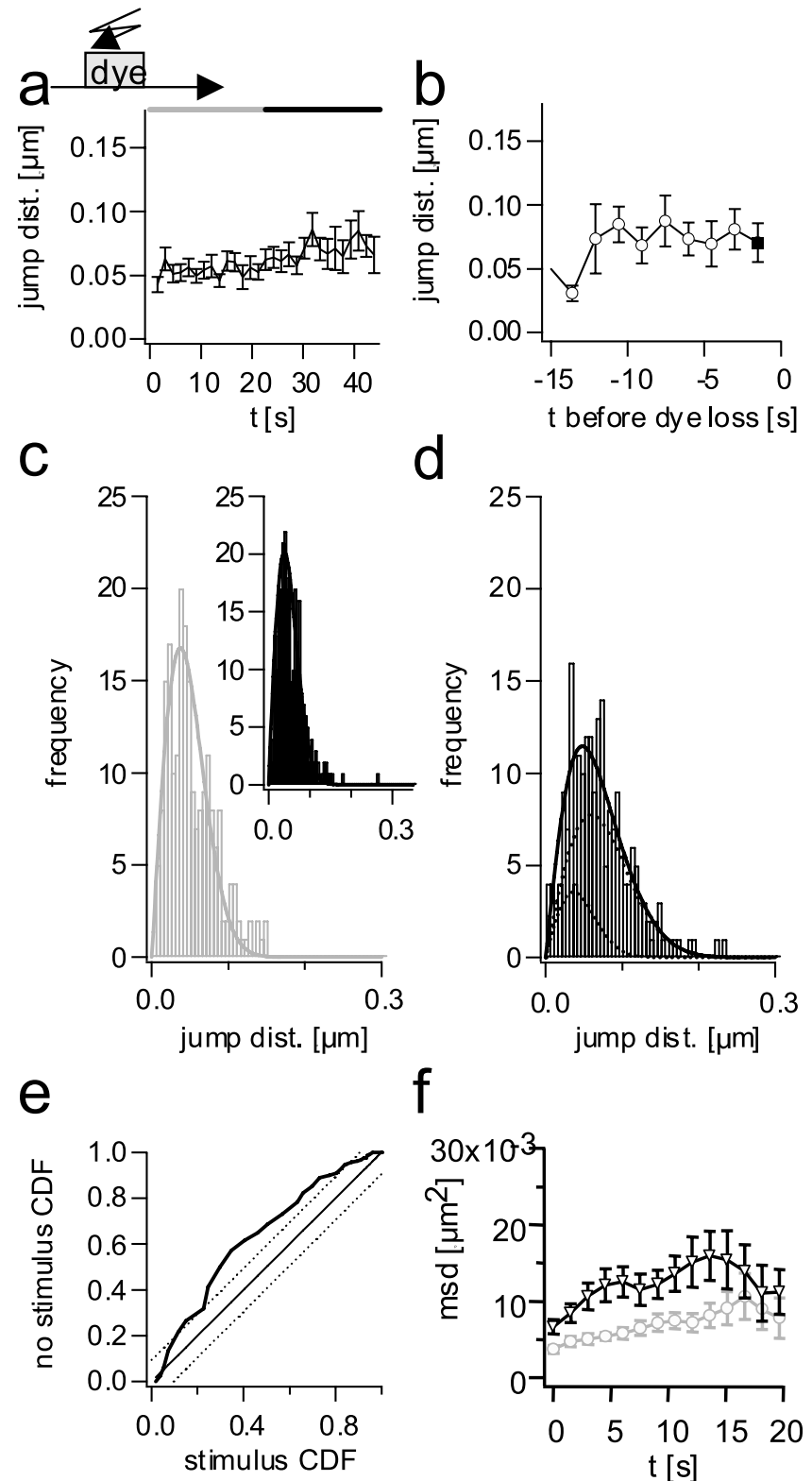

$\mathrm{f}$

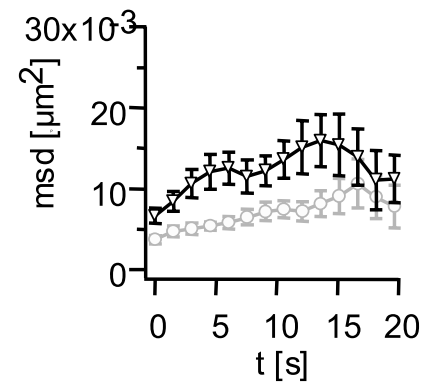

Figure 4. Quantitative mobility analysis of early endocytosed vesicles. $\boldsymbol{a}$, Frame-to-frame displacements [i.e., the distances a single vesicle moves from one frame to the next (jumps)] plotted versus time ( $\Delta$ frame $=1.512 \mathrm{~s}$ ). $\boldsymbol{b}$, Frame-to-frame displacements, time-aligned with respect to the detected dye-loss events for 10 vesicles that fused during weak stimulation. $\boldsymbol{c}, \boldsymbol{d}$, Jump frequency histogram of vesicles during resting conditions and during stimulation. The distribution in c was fit with a one-component model and in $\boldsymbol{d}$ with a two-component model. Individual components are shown in dotted and dashed lines. A two-component fit to $c$ did not return reasonable parameters. The inset in c shows the distribution for putative single vesicles in fixed cultures. $\boldsymbol{e}$, Normalized CDF calculated from c and $\boldsymbol{d}$ plotted against each other (Kolomogorv-Smirnov test). Dotted lines show the $95 \%$ confidence intervals calculated according to the K-S test with $\pm 1.36 / \sqrt{n}$ with $n=208$ frames analyzed. $\boldsymbol{f}$, Shown is the MSD for vesicles resting (gray) and during stimulation (black). Error bars indicate SEM.

reported previously (Ryan et al., 1997; Murthy and Stevens, 1998; Aravanis et al., 2003; Jordan et al., 2005), and a fit with Equation 1 revealed the intensity contribution of a single vesicle to be 98 counts, as expected. However, it showed a small intensity offset caused by a minor bleed-through of fluorescent light of FM5-95 into the green detection channel, which was determined to be 12 counts by measuring the fluorescence intensity in the green channel for synapses that failed to take up FM1-43 (Fig. $2 f$, inset) (for details, see Materials and Methods).
The double-labeling protocol of single vesicles in green and the whole recycling pool of the synapse in red gave the possibility to solve problem 1 by analyzing the mobility of vesicles and synapses simultaneously. For mobility analysis, the time series acquired between the two z-stack recordings was analyzed (Fig. 1a) (see below). As described in Materials and Methods, the redstained synapses, which varied in size and shape, were tracked with a cross-correlation algorithm and the subresolution single vesicles in the green channel with direct Gaussian fit to the fluorescent spot. Because we were only interested in studying single vesicle dynamics, we excluded all ROIs in which synapse movement was observed (for details, see Materials and Methods) (supplemental Fig. 3, available at www.jneurosci.org as supplemental material).

\section{Addressing problem 2: selection criteria from time-series recordings}

To ensure that all vesicles taken for analysis were from ROIs with similar imaging quality, we used a semiautomated selection procedure (for details, see Materials and Methods) and applied selection criteria to ROIs in the time series. The position of single vesicles in every frame of the time series was determined by fitting a 2D Gaussian. The amplitude (a measure for the intensity of the object), the offset of the amplitude (a measure for the background), and the width (a characteristic for subresolution particles) of this fit were required to lie within certain boundaries (for details, see Materials and Methods).

\section{Addressing problem 3: detecting dye-loss events and halting the tracking algorithm}

In the following, we present mobility analysis of early endocytosed vesicles studied at rest and during stimulation at $3 \mathrm{~Hz}$. For this, a stimulation protocol was time-aligned with the acquisition of the 40 images of the time series (Fig. $1 b$ ). To rule out that a vesicle was tracked although it had already fused and lost dye, it was not just sufficient to merely pick fluorescent puncta of single vesicles with similar intensity at the beginning of the time series, but also to halt the algorithm and exclude data points from vesicles that lost a major fraction of their initial intensity, because this could lower the precision of the tracking procedure. Aravanis et al. (2003) suggested that most vesicles loose $\geq 50 \%$ of their dye content when they fuse. Thus, the threshold for halting the tracking algorithm was set to $60 \%$ of the object amplitude during resting conditions. This selection criterion ensured that tracking halted, independently of what type of fusion event had occurred (partial or full release). Figure $3 a$ shows a sudden decrease in the amplitude after alignment of fit amplitudes of vesicles with respect to the time point of detected dye loss. Because FM1-43 departitions slowly from the membrane $(\sim 3$ s) (Ryan et al., 1996a; Klingauf et al., 1998), the actual fusion could have happened before the detected dye loss so that images were analyzed up to one frame (excluding this frame) before the detected intensity drop of $>40 \%$.

Our algorithm selects for those fluorescent objects that fulfill the abovementioned criteria and in which an intensity drop is detected during time-series acquisition (i.e., during arrival of the first 525 APs) (Fig. 1b). In our first set of experiments on early endocytosed vesicles, 20 were determined by the algorithm to be trackable, and Figure $3 b$ shows the distribution of dye-loss events over time for those vesicles. For early endocytosed vesicles, a dye-loss event was detected during stimulation with $3 \mathrm{~Hz}$ (frames 16-30; in total, 75 APs). Aravanis et al. (2003) reported a high 
release probability of early endocytosed vesicles. Differences between this and their study may originate from the somewhat low sample number in both studies, as well as slightly different experimental conditions.

In Figure $3 c$, the trajectories from five early endocytosed vesicles are shown as an example. The trajectory of the vesicle during resting conditions is shown in gray (frames 1-15) and during stimulation with $3 \mathrm{~Hz}$ in black (frames 16-30). Plotted are also four trajectories in which putative single vesicles were tracked in a culture after fixation with $4 \%$ paraformaldehyde. This yield is a good estimate for the tracking precision (see below) (Table 1).

\section{Mobility analysis of early endocytosed vesicles}

Because about one-half of the vesicles lost their dye content until frame 30 (after arrival of $75 \mathrm{APs}$ ), we limited our mobility analysis to those 30 frames to have sufficient data points for quantitative analysis. First, we calculated the distance of a vesicle movement from one frame to the next (Fig. 4a). The plot indicates only a very gentle increase in the frame-to-frame jump distance over time during stimulation. Next, we wanted to know whether there was a common trend in vesicle motion (for example, a higher velocity shortly before dye loss, hinting at a translocation over larger distances to the plasma membrane). For this, all vesicles in which a dye loss event was detected until frame 30 were aligned with respect to the time point of dye loss.

Interestingly, although the slow departitioning rate of FM1-43 might not allow a precise position estimate of the vesicle in the last frame before dye loss (highlighted in black), resulting in an artificially higher vesicle mobility, we could not observe a drastic change in the plot even before fusion. From this plot, we could also estimate that average vesicles did not exceed a velocity of $0.090 \mu \mathrm{m} / \mathrm{s}$.

\section{Jump frequency distribution and mean square displacement analysis}

Next, we characterized vesicle mobility using the jump frequency distribution (Crank, 1975). This type of analysis has been successfully used previously (e.g., to identify and characterize three different mobility subpopulations of U1 splicing factors in the cell nucleus) (Kues et al., 2001). For proper statistical comparison of vesicle mobility during resting conditions and stimulation, it was necessary to analyze for each vesicle the same number of frames for both conditions. This is best explained by giving two examples, which are described below.

Example 1. A dye loss was detected in frame 34. Frames 1-15 (15 frames in total) for this vesicle, in the absence, and frames 16-30 (also 15 frames in total), during which the culture was stimulated continuously at $3 \mathrm{~Hz}$, were analyzed.

Example 2. A dye loss was detected in frame 29. The analysis was thus restricted to frames up to and including frame 27. For resting conditions, frames $4-15$ were analyzed, and for stimulation, frames 16-27.

Figure $4 c$ shows the jump frequency distribution analysis for the 20 early endocytosed vesicles during resting conditions. The corresponding fit according to Equation 3 revealed one component with $D=4.31 \times 10^{-4 \frac{\mu \mathrm{m}^{2}}{\mathrm{~s}}}$ (all data are also summarized in Table 1). Even when tracking a perfectly immobile particle, jump frequency distribution analysis would yield a nonzero diffusion coefficient because of the precision of every tracking algorithm. Figure $4 c$, inset, shows the distribution for putative single vesicles that were immobilized using 4\% paraformaldehyde yielding $D=4.7 \times 10^{-4 \frac{\mu \mathrm{m}^{2}}{\mathrm{~s}}}$. Thus, during resting conditions, early endocytosed vesicles were either completely immobile or moved very slowly.

In Figure $4 d$, the same vesicles as in Figure $4 c$ were analyzed during $3 \mathrm{~Hz}$ stimulation. One can readily see that the histogram is slightly wider, indicating a mobility increase. The data could be fit with one component, which returned $D=9.37 \times 10^{-4 \frac{\mu \mathrm{m}^{2}}{\mathrm{~s}}}$. When fitting the data with two components $\mathrm{f} 1 \mathrm{stim}=0.21, \mathrm{D} 1 \mathrm{stim}=$ $4.31 \times 10-4 \mu \mathrm{m} 2 \mathrm{~s} \quad($ fixed $)$ and $f 2$ stim $=0.79$, D2stim $=$ $11.97 \times 10-4 \mu \mathrm{m} 2 \mathrm{~s}$ were obtained. For easier comparison between different loading paradigms (see below for late and spontaneously endocytosed vesicles), the first component was fixed to the D determined for the respective resting vesicles. To test whether the two components indeed described our data better, we calculated the AIC (see Materials and Methods) (Akaike, 1981), which returned $\mathrm{AIC}_{1 \mathrm{C}}=$ -774.691 for the one-component fit and $\mathrm{AIC}_{2 \mathrm{C}}=-1063.85$ for the two-component fit. Thus, it was more likely that we have two components of vesicle mobility during stimulation. Significant mobilization after stimulation was also suggested by a Kolmogorov-Smirnov (K-S) test of the two normalized cumulative distribution functions (CDFs) (Fig. 4e), which showed that the two jump frequency distributions were significantly different.

Mean square displacement (MSD) (see Materials and Methods) analysis is a powerful technique for studying single object dynamics (Saxton, 1993), because the shape of the MSD carries information about the type of mobility (Steyer et al., 1997). The MSD plots in Figure $4 f$ show, for resting conditions, a saturating 

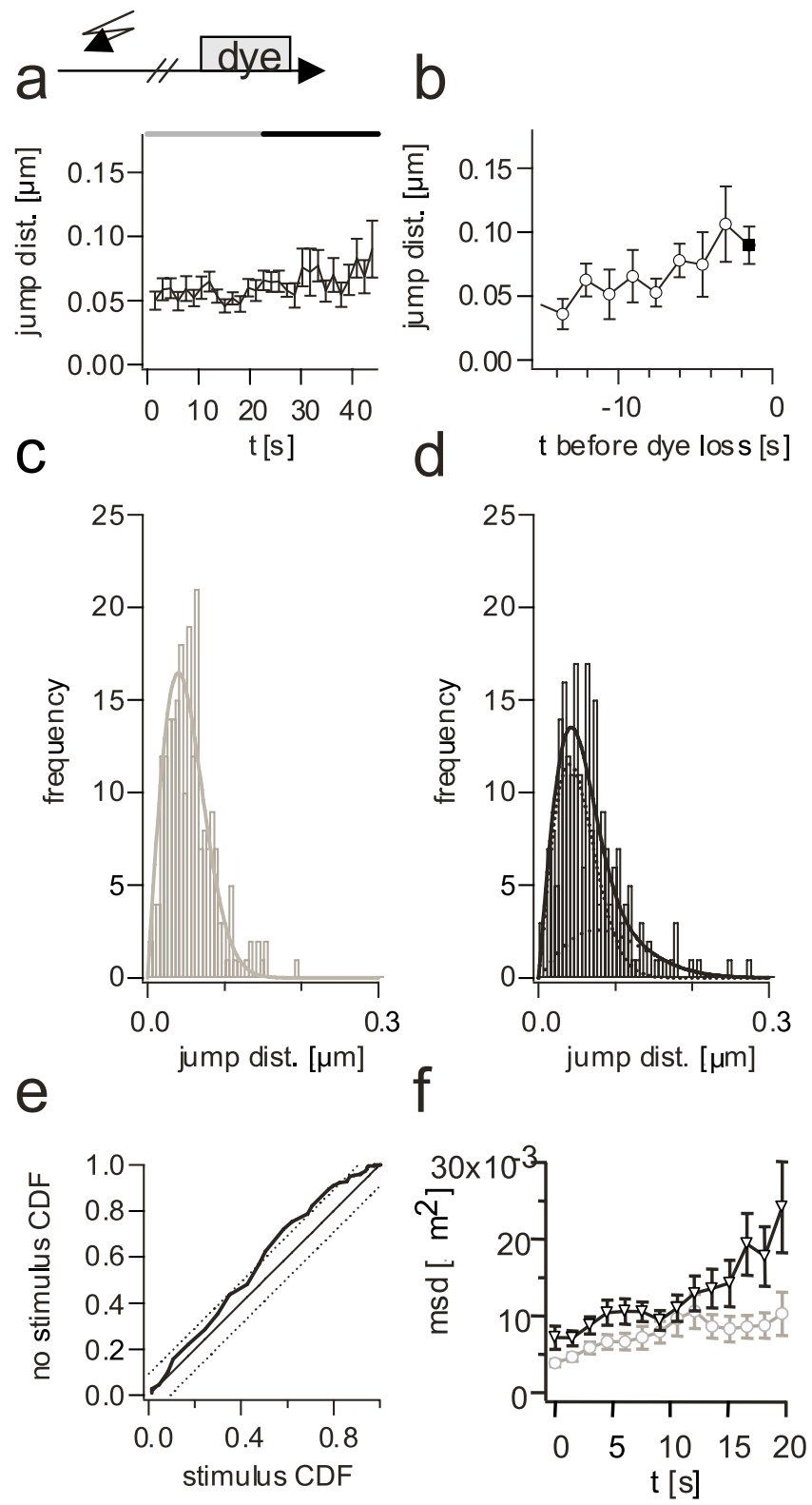

Figure 6. Quantitative mobility analysis of late endocytosed vesicles (compare with Fig. 4). $\boldsymbol{a}$, Frame-to-frame displacements plotted versus time ( $\Delta$ frame $=1.512 \mathrm{~s}$ ). $\boldsymbol{b}$, Frame-to-frame displacements time-aligned with respect to the detected dye-loss event for nine vesicles that fused during weak stimulation. $\boldsymbol{c}, \boldsymbol{d}$, Jump frequency histogram of vesicles during resting conditions and stimulation, and corresponding fits with one-component and two-component models, respectively. Individual components are shown in dotted and dashed lines. $\boldsymbol{e}$, Normalized CDF calculated from cand $\boldsymbol{d}$ plotted against each other ( $\mathrm{K}-\mathrm{S}$ test). The dotted lines show the $95 \%$ confidence intervals. $f$, MSDs for vesicles resting (gray) and during weak synaptic activity (black). Error bars indicate SEM.

curve typical for confined diffusion, and from the square root of the plateau of this plot, we obtained an upper limit for the cage radius in which mobility took place (here, $0.1 \mu \mathrm{m}$ ). The MSD plot for vesicles during stimulation was noisy, but indicated mobilization of vesicles, consistent with our previous analysis.

\section{Late endocytosed vesicles}

After characterizing the dynamics of early endocytosed vesicles, we turned now to characterizing dynamics of vesicles that were endocytosed under different conditions. We adapted a minimal staining protocol used by Murthy and Stevens (1999) to preclude that vesicles were taken up by an early mechanism. The culture was stimulated with $100 \mathrm{AP}$ at $10 \mathrm{~Hz}$, and $30 \mathrm{~s}$ after stimulation, cells were perfused with $16 \mu \mathrm{M}$ FM1-43 for $10 \mathrm{~s}$. Analysis in this and the following section was performed identically to that in the preceding section for early endocytosed vesicles. Figure $5 a$ shows the quantal single vesicle histogram, which revealed a single vesicle fluorescence of 99 counts and a bleed-through of 10 counts (Fig. $5 a$, inset). Figure $5 b$ shows the amplitude of the Gaussian fit to the fluorescent spot aligned to the detected dye-loss events, and Figure $5 c$ shows the distribution of fusion events of the 22 tracked vesicles, of which $\sim 10$ fused during the first 75 APs applied at $3 \mathrm{~Hz}$. Again, vesicles appeared to be rather immobile at rest, and slightly but significantly mobilized during stimulation (Fig. $6 a-d$, Table 1). A two-component fit to the jump frequency histogram during stimulation (Fig. $6 d$ ) was suggested by the AIC, as for early endocytosed vesicles (Table 1). The MSD plot in Figure $6 f$ shows the typical shape for confined diffusion with a maximal cage radius of $\sim 0.1 \mu \mathrm{m}$ for vesicles during resting conditions. In summary, early and late endocytosed vesicles behaved similarly with respect to their mobility and the distribution of dye-loss waiting times.

\section{Spontaneously endocytosed vesicles}

The similar results for early and late endocytosed vesicles may be an indication that these vesicles were, in fact, endocytosed by the same mechanism and recycled back to the same pools. This also would have been possible if the majority of vesicles were endocytosed spontaneously (miniature events) rather than by stimulusinduced exo-endocytotic recycling (Katz and Miledi, 1969).

To test this possibility, we aimed at staining single vesicles by applying dye for $30 \mathrm{~s}$ in the absence of any stimulation. More than 50 coverslips were measured and only very few fluorescent spots originating from vesicles or vesicle clusters could be identified. Less than two vesicles were identified to be "trackable" by our algorithm. We concluded that only stimulus-induced recycling of vesicles allowed for staining vesicles in the above protocols for early and late endocytosed vesicles, in which dye application was even limited to $10 \mathrm{~s}$.

However, it was intriguing to study the mobility of spontaneously endocytosed vesicles itself. Simply increasing the time of dye application to allow more vesicles to be spontaneously taken up, unfortunately reduced the imaging quality. Thus, we applied FM1-43 dye for $20 \mathrm{~s}$ in elevated calcium solution $\left(8 \mathrm{mM} \mathrm{Ca}^{2+}\right)$, which triggered more spontaneous fusion events in shorter time (Sara et al., 2005). Twenty-two vesicles were found to be trackable, and the results are summarized in Table 1.

Figure 7 is comparable with Figures 3 and 5, and shows the quantal histogram as well as the qualitative analysis of vesicle mobility. Figure 8 is similar to Figures 4 and 6, and shows the result of the quantitative vesicle mobility analysis. No striking difference in any of the parameters to the early and late endocytosed vesicles could be seen, and therefore, we did not find any obvious difference in vesicle mobility of early, late, and spontaneously endocytosed vesicles (see Discussion).

\section{Discussion}

We applied single particle tracking to monitor single vesicle dynamics within small hippocampal boutons. First, we showed that most of the technical problems, such as bouton movement, proper identification of single vesicles, and fluorescence loss caused by synaptic activity, can be solved by using dual labeling of single vesicles and the respective synapse. In the second part, we 
studied the mobility of individual vesicles, stained under different physiological conditions, during their lifetime until fusion.

Our dual-color staining protocol allowed us to image the whole synapse as well as a single vesicle simultaneously. By analyzing the movement of both, we could avoid contamination with synapse movement. Dual-color labeling had additional valuable advantages. Because strong synapse staining with red fluorescent dye resulted in high-contrast images, one could navigate easily to a field of view in which synapses were nicely separated from each other and the focus could be adjusted at very low laser intensity. We found this to increase data collection efficiency dramatically.

Typically, we obtained $\sim 20$ successfully tracked vesicles out of 50 experiments. We studied the mobility of early, late, and spontaneously endocytosed vesicles and found them to be surprisingly similar (Figs. 4, 6, 8; Table 1). Vesicle mobility was low and restricted (Figs. $4 f, 6 f, 8 f$, MSD plots) in the absence of stimulation. This was in line with previous data from FRAP experiments (Henkel et al., 1996; Kraszewski et al., 1996) and our recent data using FFS, in which we determined the diffusion coefficient to be as low as 5 $\times 10^{-5 \frac{\mu \mathrm{m}^{2}}{\mathrm{~s}}}$ (Jordan et al., 2005). In the previous study, we showed that already a very simple model of caged diffusion can describe the fluctuations found in the spectroscopic traces quite well, which does not exclude the possibility that more complex models can do so as well.

However, this study adds more spatial information than FRAP or FFS and we conclude that vesicle movement is not just slow, but a vesicle also remains close to its origin over a time period of at least $22 \mathrm{~s}$. Furthermore, given the finite precision of the algorithm, vesicles might be even completely immobile, such that description with a caged diffusion model may not be true for all vesicles. The term "cage" also implies some sort of molecular machinery like synapsins (Greengard et al., 1993) restricting and controlling movement, which may even not be that essential as revealed by targeted deletion of the respective genes (Rosahl et al., 1995; Ryan et al., 1996b; Godenschwege et al., 2004).

Techniques like FRAP and FFS could not be used during stimulation, because dye released during fusion compromises any quantitative analysis. Also, in particle tracking, dye release was a concern and we had to limit our mobility analysis to a time point $\sim 2$ s before dye loss to allow for complete dye departitioning from the ROI. But in many cases, we could follow the trajectory of a vesicle over tens of seconds during stimulation, which did not show high mobility of vesicles (Table 1 ). We could, however, detect a small mobility increase during stimulation using the jump frequency distribution analysis, which we could analyze assuming either one or two components. The two components were found to describe the data better (using the AIC) and might reflect two different vesicle states, with the more mobile one probably being the one leading to fusion. The second, more mobile state could be best fit with a diffusion constant twofold to fourfold higher than that under resting conditions (Table 1). In the case of free diffusion, a vesicle with such a diffusion coefficient would still need a few tens of seconds to cover a distance of one-half of a micrometer, according to the Einstein-Smoluchowski relationship $\left(\left\langle r^{2}\right\rangle=4 \cdot D t\right.$, with distance $\left.r\right)$. The following scenario is, in principle, supported by our data: vesicles do not have to move far when "called for fusion." In this case, we could further speculate that vesicles are either recycled near the AZ (Pyle et al., 2000) or are transported there shortly after endocytosis, before our data collection starts. However, FM-labeled vesicles, even those belonging to the readily releasable pool (RRP), are rather dispersed within the synaptic bouton (Schikorski and Stevens, 1997; Rizzoli and Betz, 2004), suggesting that synaptic vesicles are much more mobile in synaptic boutons than previously thought. In fact, evanescent wave microscopy of single FMlabeled vesicles in giant ribbon synapses of retinal bipolar cells revealed high mobility of vesicles close to and in between ribbons (Zenisek et al., 2000; Holt et al., 2004). In intact small synapses in hippocampal cultures, however, we found very little mobility, even during stimulation, which is difficult to reconcile with the electron microscopic data of those synapses (Schikorski and Stevens, 1997). If FM-stained vesicles are scattered throughout the bouton and are virtually immobile, even shortly before fusion, how and when are these vesicles then brought to the AZ? Our results favor a mechanism by which vesicles are activated and translocated to the AZ only 1 or $2 \mathrm{~s}$ before fusion (in our protocol, 5-10 APs). Such a mechanism is supported by studies highlighting the relevance of active transporters like myosins (Ryan, 1999; Jordan et al., 2005).

We find it very intriguing that vesicles recycled at a late step behave surprisingly similar in terms of mobility and the distribution of dye-loss events to the one recycled in an early step and commonly believed to belong to the RRP (for review, see Rizzoli and Betz, 2005). Both staining protocols have in common that they presumably label vesicles that were endocytosed last (Ryan et al., 1993). In the case of the early endocytosed vesicles, they were the first and last to be recycled at the same time, because only 5 APs were used for stimulation, whereas late endocytosed vesicles were the last ones anyhow. Previously, it has been observed that vesicles endocytosed fast after mild stimulation preferably repop- 

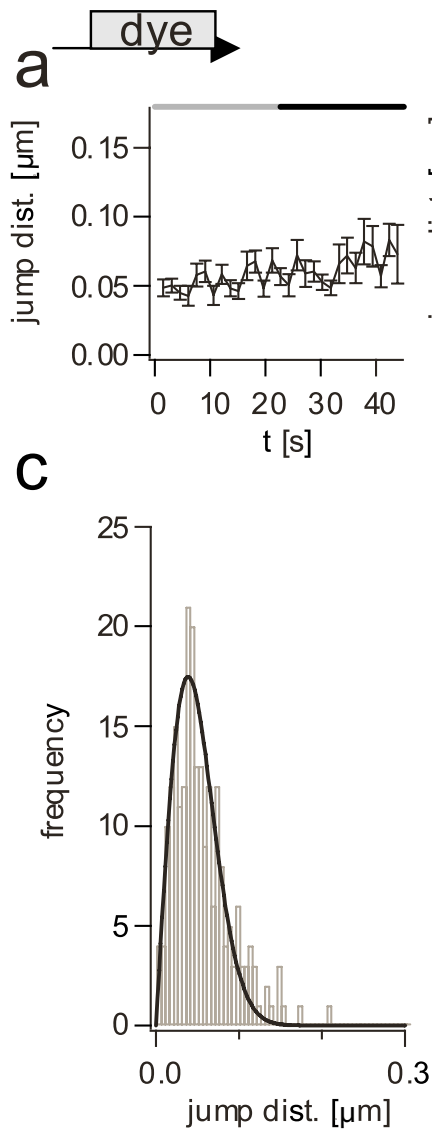

e
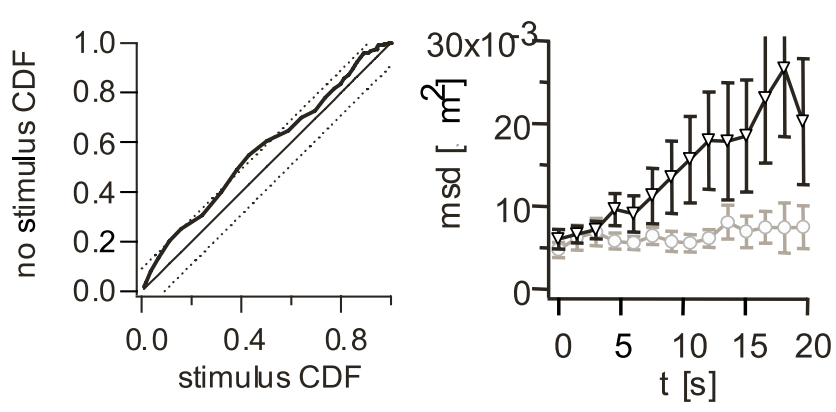

Figure 8. Quantitative mobility analysis of spontaneously endocytosed vesicles (compare with Figs. 4, 6). $\boldsymbol{a}$, Frame-to-frame displacements plotted versus time ( $\Delta$ frame $=1.512 \mathrm{~s}$ ). $\boldsymbol{b}$, Frame-to-frame displacements time-aligned with respect to the detected dye-loss event for 11 vesicles that fused during weak stimulation. $\boldsymbol{c}, \boldsymbol{d}$, Shown is the jump frequency histogram of vesicles during resting conditions and stimulation, and corresponding fits with one-component and two-component models, respectively. Individual components are shown in dotted and dashed lines. $\boldsymbol{e}$, Normalized CDF calculated from c and $\boldsymbol{d}$ plotted against each other (K-S test). The dotted lines show the 95\% confidence intervals. $f$, MSDs for vesicles resting (gray) and during weak synaptic activity (black). Error bars indicate SEM.

ulate the RRP (Richards et al., 2003; Rizzoli and Betz, 2004). Our data extend this notion and provide evidence that vesicles endocytosed last are always directed to the RRP, regardless of whether they are taken up early or late, or by weak or strong stimulation (for review, see Matthews, 2004). Perhaps these last endocytosed vesicles are brought to the edge of the cluster and therefore are less tightly bound to and integrated into the cluster, and could even be attached to actin, which is found at the periphery (Sankaranarayanan et al., 2003) and could rapidly propel those vesicles to the active zone. Such a scenario would be in line with electron microscopic data by Rizzoli and Betz (2004) and was discussed by the authors.

In our last set of experiments, we investigated spontaneously endocytosed vesicles, which are suggested by Sara et al. (2005) to belong to a different pool than the RRP (but see Prange and Murphy, 1999). In contrast to the study by Sara et al. (2005), our analysis was limited to those vesicles that clearly destained during arrival of the first 525 APs (see Materials and Methods) (Fig. 1). For these vesicles, we observed the same characteristics for early and late endocytosed vesicles [i.e., low mobility (caged slow diffusion or complete immobility) during resting conditions and only slightly increased mobility during stimulation]. However, vesicles endocytosed after stimulation may be vesicles released asynchronously (i.e., after calcium domains have collapsed, given that FM1-43 was present for seconds). Asynchronous release may involve a different set of synaptotagmins (Hui et al., 2005), maybe identical to those for spontaneous release, and therefore, both types of vesicles may be recycled by adaptor proteins different from those for synchronously released vesicles. Because, however, the contribution of asynchronous release is only $\sim 5 \%$ (Taschenberger et al., 2005), we think that our mobility analysis reflects mostly the behavior of synchronously released vesicles.

Recently, we also introduced FFS as a new tool to study vesicle mobility in hippocampal boutons (Jordan et al., 2005), but unfortunately, this technique cannot be easily applied to stimulated synapses. Although SVT yields more spatial information, we see FFS as a complementary method to SVT, because FFS experiments are less time-consuming. Furthermore, certain drug treatments might not be possible at all in SVT experiments. In preliminary experiments with okadaic acid (data not shown), which has been shown to free vesicles from the tethers (Henkel et al., 1996; Jordan et al., 2005), for example, we noticed a decrease in the imaging quality, which was of no problem in the FFS experiments on stronger stained synapses (Jordan et al., 2005) but prohibited data collection in the SVT experiments.

In summary, we successfully established single synaptic vesicle tracking in small hippocampal boutons. Using this method, it should be possible to close the gap in our understanding of the dynamics of small synaptic vesicles in small synapses, as opposed to large granule mobility in endocrine cells, where particle tracking studies are widely applied in combination with, for example, evanescent wave and/or confocal microscopy (Steyer et al., 1997; Steyer and Almers, 1999). However, our approach must be further technically advanced. One problem was the time resolution, which was a compromise between reducing strong dye bleaching and the current technical properties of our setup. Increasing the sampling rate in laser-scanning microscopy can typically be done at the cost of acquiring smaller images or by using more advanced laser-scanning technology (Tan et al., 1999). Certainly, a better fluorescent label, such as quantum dots, would greatly enhance the power of this technique. Alternatively, the spectral bandwidth for detecting green fluorescence could be increased using spectral unmixing (Neher and Neher, 2004), which would require, however, to account for nonlinear effects of the detectors in the theory (see Materials and Methods).

The method should be further developed to acquire information faster on more vesicles at a higher time resolution during higher frequency stimulation of the synapse. The future challenge will certainly be the tracking of single vesicles during the last seconds before fusion. 


\section{References}

Akaike H (1981) Likelihood of a model and information criteria. J Econom 16:3-14.

Aravanis AM, Pyle JL, Tsien RW (2003) Single synaptic vesicles fusing transiently and successively without loss of identity. Nature 423:643-647.

Betz WJ, Mao F, Bewick GS (1992) Activity-dependent fluorescent staining and destaining of living vertebrate motor nerve terminals. J Neurosci 12:363-375.

Cheezum MK, Walker WF, Guilford WH (2001) Quantitative comparison of algorithms for tracking single fluorescent particles. Biophys J 81:2378-2388.

Crank J (1975) The mathematics of diffusion. London: Clarendon.

Dunaevsky A, Tashiro A, Majewska A, Mason C, Yuste R (1999) Developmental regulation of spine motility in the mammalian central nervous system. Proc Natl Acad Sci USA 96:13438-13443.

Fischer M, Kaech S, Knutti D, Matus A (1998) Rapid actin-based plasticity in dendritic spines. Neuron 20:847-854.

Gandhi SP, Stevens CF (2003) Three modes of synaptic vesicular recycling revealed by single-vesicle imaging. Nature 423:607-613.

Godenschwege TA, Reisch D, Diegelmann S, Eberle K, Funk N, Heisenberg M, Hoppe V, Hoppe J, Klagges BR, Martin JR, Nikitina EA, Putz G, Reifegerste R, Reisch N, Rister J, Schaupp M, Scholz H, Schwarzel M, Werner U, Zars TD, et al. (2004) Flies lacking all synapsins are unexpectedly healthy but are impaired in complex behaviour. Eur J Neurosci 20:611-622.

Greengard P, Valtorta F, Czernik AJ, Benfenati F (1993) Synaptic vesicle phosphoproteins and regulation of synaptic function. Science 259:780-785.

Harata N, Ryan TA, Smith SJ, Buchanan J, Tsien RW (2001a) Visualizing recycling synaptic vesicles in hippocampal neurons by FM1-43 photoconversion. Proc Natl Acad Sci USA 98:12748-12753.

Harata N, Pyle JL, Aravanis AM, Mozhayeva M, Kavalali ET, Tsien RW (2001b) Limited numbers of recycling vesicles in small CNS nerve terminals: implications for neural signaling and vesicular cycling. Trends Neurosci 24:637-643

Heintzmann R (1999) Resolution enhancement of biological light microscopic data. PhD thesis, Rupertus Carola University.

Henkel AW, Betz WJ (1995) Staurosporine blocks evoked release of FM1-43 but not acetylcholine from frog motor nerve terminals. J Neurosci 15:8246-8258.

Henkel AW, Simpson LL, Ridge RM, Betz WJ (1996) Synaptic vesicle movements monitored by fluorescence recovery after photobleaching in nerve terminals stained with FM1-43. J Neurosci 16:3960-3967.

Heuser JE, Reese TS (1973) Evidence for recycling of synaptic vesicle membrane during transmitter release at the frog neuromuscular junction. J Cell Biol 57:315-344.

Holt M, Cooke A, Neef A, Lagnado L (2004) High mobility of vesicles supports continuous exocytosis at a ribbon synapse. Curr Biol 14:173-183.

Hui E, Bai J, Wang P, Sugimori M, Llinas RR, Chapman ER (2005) Three distinct kinetic groupings of the synaptotagmin family: candidate sensors for rapid and delayed exocytosis. Proc Natl Acad Sci USA 102:5210-5214.

Jordan R, Lemke EA, Klingauf J (2005) Visualization of synaptic vesicle movement in intact synaptic boutons using fluorescence fluctuation spectroscopy. Biophys J 89:2091-2102.

Katz B, Miledi R (1969) Spontaneous and evoked activity of motor nerve endings in calcium ringer. J Physiol (Lond) 203:689-706.

Klingauf J, Kavalali ET, Tsien RW (1998) Kinetics and regulation of fast endocytosis at hippocampal synapses. Nature 394:581-585.

Korn H, Faber DS (1991) Quantal analysis and synaptic efficacy in the CNS. Trends Neurosci 14:439-445.

Kraszewski K, Daniell L, Mundigl O, De Camilli P (1996) Mobility of synaptic vesicles in nerve endings monitored by recovery from photobleaching of synaptic vesicle-associated fluorescence. J Neurosci 16:5905-5913.

Kues T, Dickmanns A, Luhrmann R, Peters R, Kubitscheck U (2001) High intranuclear mobility and dynamic clustering of the splicing factor U1 snRNP observed by single particle tracking. Proc Natl Acad Sci USA 98:12021-12026.
Levitan ES (2004) Using GFP to image peptide hormone and neuropeptide release in vitro and in vivo. Methods 33:281-286.

Matthews G (2004) Cycling the synapse: scenic versus direct routes for vesicles. Neuron 44:223-226.

Murthy VN, Stevens CF (1998) Synaptic vesicles retain their identity through the endocytic cycle. Nature 392:497-501.

Murthy VN, Stevens CF (1999) Reversal of synaptic vesicle docking at central synapses. Nat Neurosci 2:503-507.

Neher RA, Neher E (2004) Applying spectral fingerprinting to the analysis of FRET images. Microsc Res Tech 64:185-195.

Prange O, Murphy TH (1999) Correlation of miniature synaptic activity and evoked release probability in cultures of cortical neurons. J Neurosci 19:6427-6438

Pyle JL, Kavalali ET, Piedras-Renteria ES, Tsien RW (2000) Rapid reuse of readily releasable pool vesicles at hippocampal synapses. Neuron 28:221-231

Qian H, Sheetz MP, Elson EL (1991) Single particle tracking, analysis of diffusion and flow in two-dimensional systems. Biophys J 60:910-921.

Rea R, Li J, Dharia A, Levitan ES, Sterling P, Kramer RH (2004) Streamlined synaptic vesicle cycle in cone photoreceptor terminals. Neuron 41:755-766.

Richards DA, Guatimosim C, Rizzoli SO, Betz WJ (2003) Synaptic vesicle pools at the frog neuromuscular junction. Neuron 39:529-541.

Rizzoli SO, Betz WJ (2004) The structural organization of the readily releasable pool of synaptic vesicles. Science 303:2037-2039.

Rizzoli SO, Betz WJ (2005) Synaptic vesicle pools. Nat Rev Neurosci 6:57-69.

Rosahl TW, Spillane D, Missler M, Herz J, Selig DK, Wolff JR, Hammer RE, Malenka RC, Sudhof TC (1995) Essential functions of synapsins I and II in synaptic vesicle regulation. Nature 375:488-493.

Ryan TA (1999) Inhibitors of myosin light chain kinase block synaptic vesicle pool mobilization during action potential firing. J Neurosci 19:1317-1323.

Ryan TA, Reuter H, Wendland B, Schweizer FE, Tsien RW, Smith SJ (1993) The kinetics of synaptic vesicle recycling measured at single presynaptic boutons. Neuron 11:713-724.

Ryan TA, Smith SJ, Reuter H (1996a) The timing of synaptic vesicle endocytosis. Proc Natl Acad Sci USA 93:5567-5571.

Ryan TA, Li L, Chin LS, Greengard P, Smith SJ (1996b) Synaptic vesicle recycling in synapsin I knock-out mice. J Cell Biol 134:1219-1227.

Ryan TA, Reuter H, Smith SJ (1997) Optical detection of a quantal presynaptic membrane turnover. Nature 388:478-482.

Sankaranarayanan S, Atluri PP, Ryan TA (2003) Actin has a molecular scaffolding, not propulsive, role in presynaptic function. Nat Neurosci 6:127-135.

Sara Y, Virmani T, Deak F, Liu X, Kavalali ET (2005) An isolated pool of vesicles recycles at rest and drives spontaneous neurotransmission. Neuron 45:563-573.

Saxton MJ (1993) Lateral diffusion in an archipelago, single-particle diffusion. Biophys J 64:1766-1780.

Schikorski T, Stevens CF (1997) Quantitative ultrastructural analysis of hippocampal excitatory synapses. J Neurosci 17:5858-5867.

Steyer JA, Almers W (1999) Tracking single secretory granules in live chromaffin cells by evanescent-field fluorescence microscopy. Biophys 76:2262-2271.

Steyer JA, Horstmann H, Almers W (1997) Transport, docking and exocytosis of single secretory granules in live chromaffin cells. Nature 388:474-478.

Sudhof TC (2000) The synaptic vesicle cycle revisited. Neuron 28:317-320.

Tan YP, Llano I, Hopt A, Wurriehausen F, Neher E (1999) Fast scanning and efficient photodetection in a simple two-photon microscope. J Neurosci Methods 92:123-135.

Taschenberger H, Scheuss V, Neher E (2005) Release kinetics, quantal parameters and their modulation during short-term depression at a developing CNS synapse. J Physiol (Lond) 568:513-537.

Thompson RE, Larson DR, Webb WW (2002) Precise nanometer localization analysis for individual fluorescent probes. Biophys J 82:2775-2783.

Zenisek D, Steyer JA, Almers W (2000) Transport, capture and exocytosis of single synaptic vesicles at active zones. Nature 406:849-854. 